\title{
Ecoendoscopia en la evaluación de las lesiones subepiteliales duodenales
}

\section{Endoscopic Ultrasound for Evaluating Subepithelial Duodenal Lesions}

Martín Alonso Gómez Zuleta, MD. ${ }^{1}$

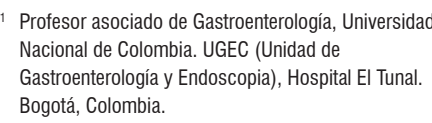

Profesor asociado de Gastroenterología, Universidad Nacional de Colombia. UGEC (Unidad de

Gastroenterología y Endoscopia), Hospital El Tunal. Bogotá, Colombia.

Fecha recibido: $27-10-14$

Fecha aceptado: 06-04-15

\begin{abstract}
Resumen
Las lesiones o tumores subepiteliales (TSE) son raros. Se considera que en 1 de cada 300 endoscopias puede encontrarse un TSE, lo que corresponde al $0,36 \%$ de las endoscopias. De estos, solo el $10 \%$ se ubican en el duodeno, lo que proporciona una idea de lo infrecuente que es este hallazgo. Al realizar la endoscopia y detectar un TSE en el duodeno, inicialmente se describirá su tamaño, forma, color, movilidad, pulsación y finalmente la consistencia, la cual puede evaluarse con la pinza de biopsia cerrada. Esto permitirá detectar si es quístico, firme o si tiene el "signo de la almohada", que es altamente sugestivo de lipoma. En caso contrario, está indicada la realización de una ecoendoscopia, especialmente si el tumor es mayor de $1 \mathrm{~cm}$.
\end{abstract}

\section{Palabras claves}

Subepitelial, duodeno, ecoendoscopia, GIST, neuroendocrino.

\section{Abstract}

Subepithelial lesions (SE) are rare. They are found in only one out of every 300 endoscopies $(0.36 \%)$. Of these, only $10 \%$ are located in the duodenum. When endoscopy does detect a subepithelial lesion in the duodenum it is important to make an initial description of its size, shape, color, mobility, and consistency to touch. Next, a closed biopsy sample must be taken with pincers and analyzed to detect whether it is a cyst and whether it has a pillow sign which is highly suggestive of lipoma. When the lesion is neither a cyst nor a lipoma, endoscopic ultrasound is indicated, especially if the lesion is larger than one centimeter.

\section{Keywords}

Subepithelial, duodenum, EUS, GIST, neuroendocrine.

\section{INTRODUCCIÓN}

Las tumores subepiteliales (TSE) del tracto digestivo superior son poco frecuentes. Se considera que en solo 1 de cada 300 endoscopias puede encontrarse una TSE, lo que corresponde al 0,36\% de las endoscopias (1). Estudios recientes han mostrado que el hallazgo de estos tumores es un indicador de calidad para la detección temprana del cáncer gástrico (2). La evidencia que hay en la literatura sobre los TSE es muy pobre y la mayoría se basa en serie de casos o la opinión de expertos. Una serie reciente reunió 129 lesiones subepiteliales, de las cuales 98 se ubicaron en el estómago, 14 en el esófago y solo 11 en el duodeno $(8,5 \%)$ (3). En general se considera que la mayoría de los TSE son asintomáticos, y el $60 \%$ se ubica en el estómago; el 30\%, en el esófago; y $10 \%$, en el duodeno (4-6).

\section{¿CÓMO ENFOCAR ESTOS TUMORES?}

Cuando se realiza una endoscopia y se detecta un TSE en el duodeno, en ese mismo momento puede iniciarse su evaluación. Inicialmente se describirá su tamaño, forma, color, 
movilidad, pulsación y finalmente la consistencia, la que es posible evaluar con la pinza de biopsia cerrada. Esto permitirá detectar si es quística, firme o si tiene el "signo de la almohada”, que es altamente sugestivo de lipoma. Si la lesión tiene ligera irregularidad de la mucosa y una depresión central, es sugestiva de un páncreas ectópico. Usualmente los quistes o várices tienen una mucosa lisa y simétrica y los tumores del estroma gastrointestinal (GIST) pueden presentar una ligera ulceración y son firmes y móviles (7).

El siguiente paso que debe llevarse a cabo es la evaluación de la lesión con una técnica de imagen, para lo cual la endosonografía o el ultrasonido endoscópico (USE) es la técnica de elección que ha desplazado a las otras técnicas. Este examen está indicado solo si la lesión mide más de $1 \mathrm{~cm}$; si no, se recomienda control endoscópico en 1 año (8). La precisión del USE para diferenciar una compresión extraluminal de un tumor subepitelial es superior al 95\%, mucho mejor que otras técnicas de imagen como la ecografía convencional o la tomografía axial computarizada (TAC), las cuales ya ni se mencionan en la literatura actual $(9,10)$.

Nosotros proponemos evaluar las lesiones duodenales desde el punto de vista ecoendoscópico, de la siguiente manera: ubique la lesión en la ecocapa correspondiente y luego evalúe si es hipoecoica, isoecoica o hiperecoica.

\section{Ecocapa 2}

Corresponde a la parte más profunda de la mucosa o a la lámina propia y a la muscular mucosa. Las lesiones que se originan en esta capa son poco frecuentes y generalmente hipoecoicas.

\section{Lesiones hipoecoicas}

La mayoría de los tumores que se originan en esta capa es muscular, usualmente leiomiomas, aunque con mayor frecuencia se ubican en la capa muscular propia ( $4{ }^{a}$ capa).

En esta capa también podemos encontrar los tumores neuroendocrinos (TNE), que usualmente crecen en la $2 .^{a}$ capa y se extiende a la ecocapa 3. En estos tumores característicamente podemos ver en la mucosa un aspecto eritematoso o reticular.

\section{Lesiones anecoicas}

Ocasionalmente podemos observar que en esta capa se originan quistes de inclusión o de retención.

\section{Ecocapa 3}

Esta capa es una banda de tejido que se ve hiperecogénica a la endosonografía y de la cual se pueden originar numerosos tumores.

\section{Lesiones hiperecogénicas}

La lesión que más frecuentemente encontramos en esta capa es el lipoma (figura 1) y se caracteriza por ser una lesión hiperecoica homogénea que usualmente tiene el llamado "signo de la almohada" cuando la presionamos con la pinza de endoscopia. Los hamartomas de las glándulas de Brunner también son lesiones hiperecoicas de la 3. ${ }^{a}$ capa, aunque a diferencia de los lipomas pueden encontrarse también en la 2. ${ }^{a}$ capa, son homogéneos y en su interior puede observarse una imagen anecoica correspondiente a un ducto dilatado.

Los neurofibromas suelen ser hiperecogénicos y se originan en la submucosa o en la muscular propia.

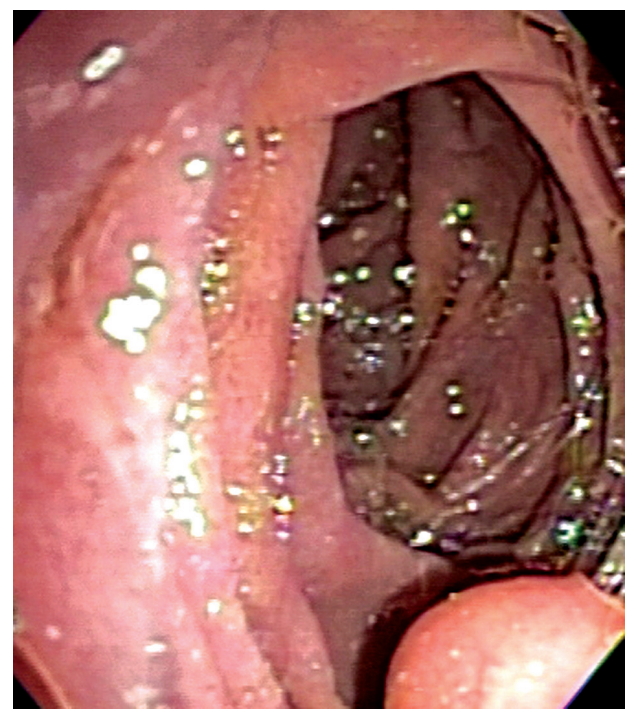

Figura 1. En la esquina inferior derecha se observa la típica imagen endoscópica de una lesión subepitelial, la cual correspondía a un lipoma.

\section{Lesiones hipoecoicas}

Una lesión hipoecoica en esta capa puede corresponder a un páncreas ectópico o aberrante. Usualmente estas lesiones son heterogéneas y ocasionalmente se puede observar una estructura ductal anecoica en el centro que puede corresponder a un conducto. En la endoscopia se observa una lesión con el centro deprimido y aunque pueden estar en el duodeno, la mayoría se localiza en el antro.

También podemos encontrar lesiones hipoecoicas que corresponden a TNE, aunque no son TSE propiamente dichos puesto que su naturaleza no es mesenquimal sino neuroendocrina. Suelen ser pequeños (menores de $1 \mathrm{~cm}$ ), hipoecogénicos (aunque más ecogénicos que la muscular) y se asientan en la mucosa profunda (capa 2), por lo que su estudio histológico suele ser posible mediante biopsias.

Los linfomas gástricos también se pueden presentar como lesiones hipo o hiperecoicas que dependen de la 
submucosa. Otras lesiones menos frecuentes que son hipoecoicas y que dependen de la $4 .^{\text {a }}$ capa son los neurofibromas y las y metástasis, que son muy raras en el duodeno.

\section{Lesiones anecoicas}

Cuando observamos lesiones anecoicas en esta capa, debemos pensar en estructuras vasculares o quistes. Para diferenciarlos, se les coloca la señal del eco-Doppler, que ayuda a evaluar si hay flujo. Los quistes que se observan dentro de la pared del tracto gastrointestinal son generalmente benignos y pueden ser quistes de inclusión, duplicación o retención.

\section{Ecocapa 4}

Esta capa corresponde a la muscular propia, por lo que la mayoría de los tumores en esta capa son de origen muscular.

\section{Lesiones hiperecoicas}

Son lesiones muy raras pero pueden corresponder a linfomas, tumores neurogénicos o metástasis.

\section{Lesiones hipoecoicas}

La mayoría de los tumores de esta capa corresponde a GIST. Aunque estos son más frecuentes en el estómago, también pueden presentarse en el duodeno (figuras $2 \mathrm{y}$ 3). Otras lesiones que pueden tener esta apariencia son las metástasis (pulmón o seno) y el tumor del glomus y los schwannomas. Los linfomas también pueden afectar esta capa pero generalmente asociado con el compromiso de las capas más superficiales.

Debido a que la correlación de la imagen ecoendoscópica con el resultado histológico final no es óptima (11), en nuestra unidad utilizamos una descripción alfanumérica para colocar el diagnostico cuando evaluamos las lesiones subepiteliales. La primera letra corresponde a la "C", que indica la capa seguida de un numero de acuerdo con la ubicación de la lesión (1 a 5). Luego ponemos la letra "E", que corresponde a la ecogenicidad que tiene la lesión ( 0 : anecoica; 1: hipoecoica; 2: isoecoica; 3 : hiperecoica), de tal forma que si en el diagnóstico colocamos "C4E1", se tratará de una lesión de la capa muscular que es hipoecoica, y en el diagnóstico diferencial puede haber GIST, leiomiomas o incluso el schwannoma.

La historia natural de los TSE depende del tipo histológico. Si se trata de una lesión sugestiva de ser benigna como el lipoma, los hamartomas de Brunner o el páncreas ectópico, usualmente tienden a no modificarse en el tiempo y no requieren seguimiento; no obstante, si se trata de tumores con potencial maligno, como el GIST, hay que seguirlos. Un estudio siguió a 49 pacientes con GIST menores de $3 \mathrm{~cm}$ durante un promedio de 30 meses y encontró que solo 5 pacientes mostraron un incremento del $25 \%$ en el tamaño, por lo cual los GIST fueron resecados (11). De otro lado, los TNE tienden a crecer y hacer metástasis, por lo que todos deben ser resecados independientemente de su tamaño. Un estudio japonés evaluó los tumores que ya tenían metástasis y encontró que en promedio medían solo $14,5 \mathrm{~mm}(12)$.

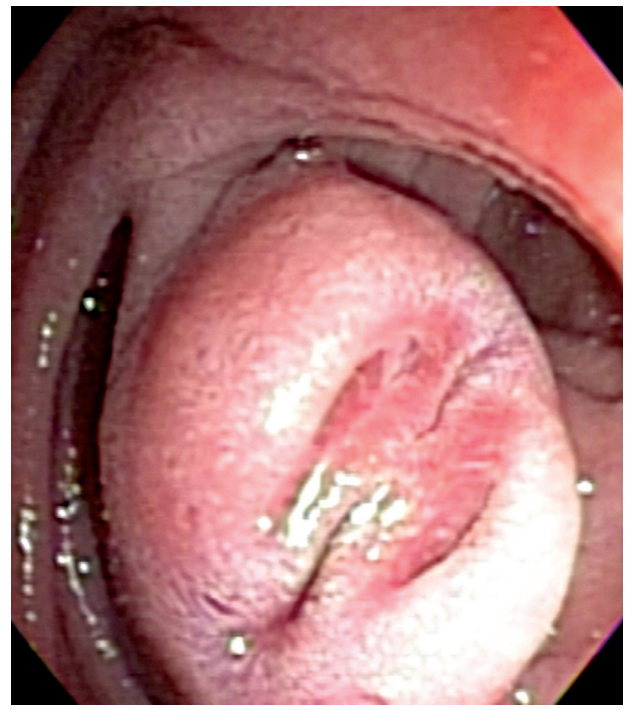

Figura 2. Observe esta imagen subepitelial con centro ulcerado en la segunda porción del duodeno, la cual fue enviada para ecoendoscopia.

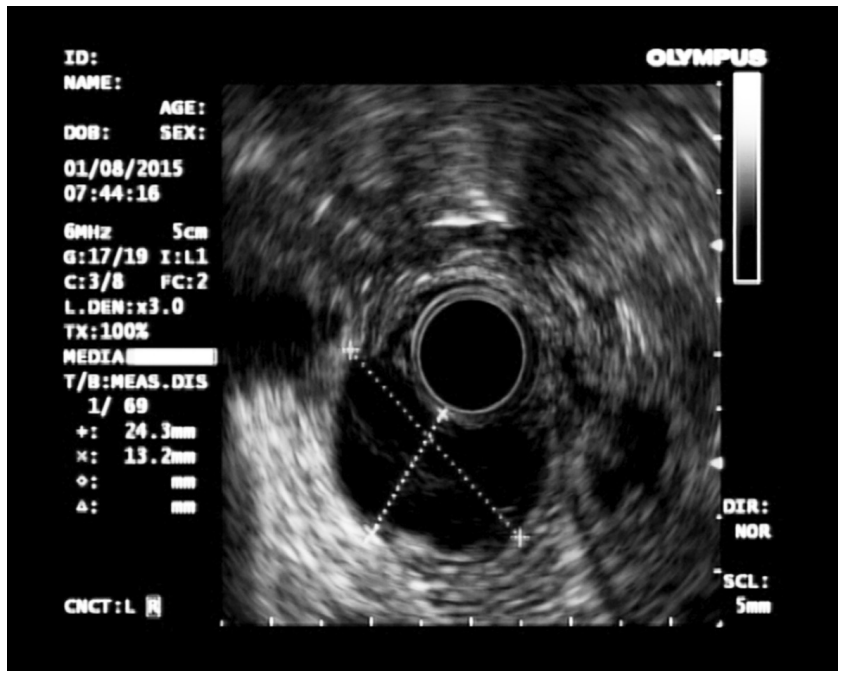

Figura 3. La ecoendoscopia muestra una lesión hipoecoica de la 4 . $^{\mathrm{a}}$ capa, que correspondía a un GIST.

\section{¿CUÁNDO REALIZAR LA PUNCIÓN DEL TSE?}

No hay un consenso en la literatura de cuándo realizar la punción del TSE y menos del duodeno. Aunque el riesgo de la misma es bajo, la producción o el rendimiento no es 
óptimo, sobre todo con las lesiones pequeñas. No existe un estudio específico de punción en lesiones duodenales; sin embargo, un trabajo de Brugge y colaboradores (13) mostró que el rendimiento de la ecoendoscopia sin la punción en lesiones menores de $2 \mathrm{~cm}$ es de solo el $45 \%$ cuando se comparó con la histología como patrón de oro.

En general se considera que la punción pudiera estar indicada en lesiones de la capa muscular propia, especialmente si son hipoecoicas y aquellas de tamaño mayor de 2 $\mathrm{cm}$, ya que se ha visto que lesiones de otras capas y que no son hipoecoicas es poco probable que sean malignas; sin embargo, en otro artículo del mismo autor se demostró que la producción de la punción del GIST del duodeno es muy mala si se compara con los del estómago (14).

El rendimiento de la punción en las lesiones subepiteliales varía de un $43,3 \%$ a un 78,4\% $(15,16)$. La producción de biopsia sobre biopsia en lesiones subepiteliales es del 17\%$38 \%$ (17). En la serie ya mencionada de Buscaglia y colaboradores (3) sobre 129 lesiones subepiteliales, se comparó el rendimiento de las biopsia jumbo con la punción, encontrando que ésta tenía en general un rendimiento similar $(58,9 \%$ versus $45,1 \%)$, pero era mejor para la biopsia jumbo cuando la lesión estaba en la $3 .{ }^{a}$ capa $(65,1 \%$ versus $35,5 \%)$ y peor cuando estaba en la $4 .{ }^{a}$ capa $(40,0 \%$ versus $57,1 \%)$. Infortunadamente el $34,9 \%$ de los pacientes tuvo sangrado significativo con la biopsia. Este trabajo sugiere que para las lesiones de la 3. ${ }^{a}$ capa deberíamos utilizar biopsia jumbo y para las de la $4 .{ }^{a}$ capa, punción con aguja fina. Otro trabajo reciente del grupo de Costamaña (18) realizó punción con aguja fina y un nuevo ecoendoscopio frontal en 121 pacientes con lesiones subepiteliales (13, en el esófago; 96, en el estómago; 10 , en duodeno; y 2 , en recto), que mostró un excelente rendimiento de la punción incluso en lesiones del duodeno, con una sensibilidad de 93,4\% (113/121) y una especificidad del $100 \%$. Lo anterior sugiere que la punción en las lesiones duodenales podría mejorarse con este equipo, ya que permite una aproximación más adecuada que con los equipos convencionales.

La punción es muy segura y tiene un bajo riesgo. Se recomienda el uso de antibióticos profilácticos solo en las lesiones quísticas. El paciente debe estar en observación por lo menos 1 hora después del procedimiento. Un estudio reunió 1135 pacientes que fueron puncionados por TSE en 219 hospitales. Las lesiones estaban ubicadas así: 73,5\%, en el estómago; $13,4 \%$, en el esófago; $8,2 \%$, en el duodeno; y 4,9\%, en otros sitios. De las 93 lesiones duodenales, 6 eran GIST. Este trabajo mostró que solo 5 pacientes $(0,44 \%)$ presentaron sangrado severo que requirió transfusión o tratamiento endoscópico; uno de ellos era por una lesión duodenal. No se presentaron perforaciones y únicamente 1 paciente $(0,09 \%)$ murió en los primeros 30 días de la punción, aunque no asociado con sangrado o perforación (19).

\section{¿CUÁNDO, EN QUIÉN Y CÓMO DEBEN TRATARSE LOS TSE?}

El tratamiento de los TSE depende de la sospecha de malignidad o de si las lesiones son sintomáticas. Se considera que las lesiones duodenales benignas, tales como el lipoma, el páncreas ectópico y los hamartomas de Brunner, no requieren tratamiento si no están produciendo síntomas como sangrado u obstrucción. Por el contrario, las lesiones con potencial maligno, como el TNE, los linfomas o el GIST, requieren tratamiento. Los pacientes con TNE duodenales menores de $1 \mathrm{~cm}$ pueden ser tratados con resección endoscópica $(20,21)$ con gran efectividad y seguridad, la cual puede llevarse a cabo con una asa o con la colocación de una banda. Los tumores que se encuentran entre 1 y $2 \mathrm{~cm}$ podrían resecarse con técnicas quirúrgicas avanzadas de resección en cuña que limitan la extensión de la cirugía o con disección de la submucosa, dependiendo de la experiencia del centro encargado (22). Las lesiones mayores de $2 \mathrm{~cm}$ deben ser manejadas de forma quirúrgica con linfadenectomía. La técnica dependerá de la ubicación y de la experiencia (23).

Los GIST menores de $2 \mathrm{~cm}$ y asintomáticos podrían observarse cada 6 a 12 meses con endoscopia, y cada año con ecoendoscopia (24), o podrían puncionarse para establecer su riesgo de malignidad si se evidencian irregularidad o espacios quísticos en su interior. De acuerdo con el resultado de la patología, deben vigilarse o someterse a cirugía. Los GIST mayores de $2 \mathrm{~cm}$ deberían ser llevados a cirugía por el riesgo de malignidad (25).

\section{¿CUÁL ES EL SEGUIMIENTO DE LOS TSE?}

Las lesiones sospechosas de GIST menores de $2 \mathrm{~cm}$ y asintomáticas deben someterse a seguimiento cada año con ecoendoscopia y cada 6 meses con endoscopia. Si las lesiones tienen bordes irregulares o espacios quísticos en su interior, deben puncionarse para establecer su pronóstico (24). Todos los TNE del duodeno se resecarse y las lesiones benignas no ameritan seguimiento porque es improbable que cambien.

La decisión del manejo quirúrgico o endoscópico en los TSE depende del tipo de lesión y su tamaño. Si la lesión es un TNE, debe resecarse endoscópicamente cuando sea menor de $1 \mathrm{~cm}$; si está entre 1 y $2 \mathrm{~cm}$, puede realizarse una disección submucosa, si es factible (26), o una resección laparoscópica en cuña (22). Las lesiones mayores de $2 \mathrm{~cm}$ deben resecarse por cirugía con linfadenectomía (23).

De otro lado, si los GIST son menores de $2 \mathrm{~cm}$, podrían observarse o puncionarse para conocer el índice de proliferación Ki-67 y establecer el pronóstico. Sin embargo, las lesiones mayores de $2 \mathrm{~cm}$ deben someterse a cirugía dado el 
riesgo de malignidad (24). Cada día se reportan más series de casos de lesiones tipo GIST que han sido resecadas de forma endoscópica a pesar de que la lesión está ubicada en la 4. ${ }^{a}$ capa o muscular propia, lo que en el futuro puede ser una muy buena opción de tratamiento (27).

En resumen, si nos enfrentamos a una lesión subepitelial y es menor de $1 \mathrm{~cm}$, no amerita ecoendoscopia sino control endoscópico en 1 año. Si la lesión es mayor, es obligatorio realizar una endosonografía. Si la lesión es pequeña y depende de la ecocapa 1, 2 o 3 , se puede observar en 1 año. Si la lesión es grande $(2-3 \mathrm{~cm})$, especialmente si es hipoecoica o se ubica en la 4. ${ }^{\text {a }}$ capa, es ideal hacer una punción guiada por ecoendoscopia. Si se establece que se trata de una lesión benigna, puede realizarse un control a los 6 meses sin punción; si no ha crecido, no necesitaría más control, aunque si es maligna o tiene algún potencial maligno se recomienda tratamiento. Si la lesión es mayor de $3 \mathrm{~cm}$, debe resecarse de forma endoscópica o quirúrgica sin necesidad de punción.

\section{REFERENCIAS}

1. Sonnenberg A, Amorosi SL, Lacey MJ, et al. Patterns of endoscopy in the United States: analysis of data from the Centers for Medicare and Medicaid Services and the National Endoscopic Database. Gastrointest Endosc. 2008;67(3):489-96.

2. Park CH, Kim B, Chung H, Lee H, Park JC, Shin SK, et al. Endoscopic quality indicators for esophagogastroduodenoscopy in gastric cancer screening. Dig Dis Sci. 2015;60(1):38-46.

3. Buscaglia J, Nagula S, Jayaraman V, Robbins D, et al. Diagnostic yield and safety of jumbo biopsy forceps in patients with subepithelial lesions of the upper and lower GI tract. Gastrointest Endosc. 2012;75:1147-52.

4. Papanikolaou IS, Triantafyllou K, Kourikou A, et al. Endoscopic ultrasonography for gastric submucosal lesions. World J Gastrointest Endosc. 2011;3(5):86-94.

5. Muenst $S$, Thies $S$, Went $P$, et al. Frequency, phenotype, and genotype of minute gastrointestinal stromal tumors in the stomach: an autopsy study. Hum Pathol. 2011;42(12):1849-54.

6. Jenssen C, Dietrich CF. Endoscopic ultrasound in subepithelial tumors of the gastrointestinal tract. En: Dietrich $\mathrm{CF}$, editor. Endoscopic ultrasound: an introductory manual and atlas. New York: Thieme; 2006. p. 121-54.

7. Souquet JC, Bobichon R. Role of endoscopic ultrasound in the management of submucosal tumours in the esophagus and stomach. Acta Endoscop. 1996;26:307-12.

8. Hwang JH, Saunders MD, Rulyak SJ, et al. A prospective study comparing endoscopy and EUS in the evaluation of GI subepithelial masses. Gastrointest Endosc. 2005;62(2):202-8.

9. Boyce GA, Sivak Jr. MV, Rosch T, et al. Evaluation of submucosal upper gastrointestinal tract lesions by endoscopic ultrasound. Gastrointest Endosc. 1991;37:449-54.
10. Caletti G, Zani L, Bolondi L, et al. Endoscopic ultrasonography in the diagnosis of gastric submucosal tumor. Gastrointest Endosc. 1989;35:413-8.

11. Bruno M, Carucci P, Repici A, Pellicano R, Mezzabotta $\mathrm{L}$, Goss $\mathrm{M}$, et al. The natural history of gastrointestinal subepithelial tumors arising from muscularis propria: an endoscopic ultrasound survey. J Clin Gastroenterol. 2009;43(9):821-5.

12. Soga J. Early-stage carcinoids of the gastrointestinal tract: an analysis of 1914 reported cases. Cancer. 2005;103:1587-95.

13. Karaca C, Turner BG, Cizginer S, Forcione D, Brugge W. Accuracy of EUS in the evaluation of small gastric subepithelial lesions. Gastrointest Endosc. 2010;71(4):722-7.

14. Sepe PS, Moparty B, Pitman MB, Saltzman JR, Brugge WR. EUS-guided FNA for the diagnosis of GI stromal cell tumors: sensitivity and cytologic yield. Gastrointest Endosc. 2009;70(2):254-61.

15. Mekky MA, Yamao K, Sawaki A, et al. Diagnostic utility of EUS-guided FNA in patients with gastric submucosal tumors. Gastrointest Endosc. 2010;71:913-9.

16. Hoda KM, Rodriquez SA, Faigel DO. EUS-guided sampling of suspected GI stromal tumors. Gastrointest Endosc. 2009;69:1218-23.

17. Sepe PS, Moparty B, Pitman MB, et al. EUS-guided FNA for the diagnosis of GI stromal cell tumors: sensitivity and cytologic yield. Gastrointest Endosc. 2009;70:254-61.

18. Larghi A, Fuccio L, Chiarello G, Attili F, Vanella G, Paliani $\mathrm{GB}$, et al. Fine-needle tissue acquisition from subepithelial lesions using a forward-viewing linear echoendoscope: Endoscopy. 2014;46(1):39-45.

19. Hamada T, Yasunaga $H$, Nakai $Y$, Isayama $H$, Horiguchi $H$, Matsuda S, et al. Rarity of severe bleeding and perforation in endoscopic ultrasound-guided fine needle aspiration for submucosal tumors. Dig Dis Sci. 2013;58(9):2634-8.

20. Waisberg J, Joppert-Netto G, Vasconcellos C, Sartini GH, Miranda LS, Franco MI. Carcinoid tumor of the duodenum: a rare tumor at an unusual site. Case series from a single institution. Arq Gastroenterol. 2013;50(1):3-9.

21. Tai WP, Yue H. Endoscopic mucosa resection of a duodenum carcinoid tumor of $1.2 \mathrm{~cm}$ diameter: a case report. Med Oncol. 2009;26:319-21.

22. Tsujimoto H, Ichikura T, Nagao S, Sato T, Ono S, Aiko S, et al. Minimally invasive surgery for resection of duodenal carcinoid tumors: endoscopic full-thickness resection under laparoscopic observation. Surg Endosc. 2010;24:471-5.

23. Zyromski NJ, Kendrick ML, Nagorney DM, Grant CS, Donohue JH, Farnell MB, et al. Duodenal carcinoid tumors: how aggressive should we be? J Gastrointest Surg. 2001;5:588-93.

24. Iorio N, Sawaya RA, Friedenberg FK. Review article: the biology, diagnosis and management of gastrointestinal stromal tumours. Aliment Pharmacol Ther. 2014;39(12):1376-86.

25. Chok AY, Koh YX, Ow MY, Allen JC Jr, Goh BK. A systematic review and meta-analysis comparing pancreaticoduodenectomy versus limited resection for duodenal gastrointestinal stromal tumors. Ann Surg Oncol. 2014;21(11)3429-38. 
26. Matsumoto S, Miyatani H, Yoshida Y, Nokubi M. Duodenal carcinoid tumors: 5 cases treated by endoscopic submucosal dissection. Gastrointest Endosc. 2011;74:1152-6.
27. Huang ZG, Zhang XS, Huang SL, Yuan XG. Endoscopy dissection of small stromal tumors emerged from the muscularis propria in the upper gastrointestinal tract: Preliminary study. World J Gastrointest Endosc. 2012;4(12):565-70. 